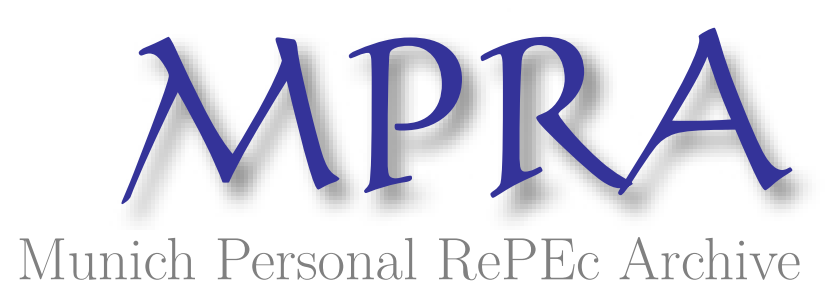

Modeling Path Dependent Counterparty Credit Risk

Zhou, Richard

Wells Fargo Bank

8 January 2015

Online at https://mpra.ub.uni-muenchen.de/61354/

MPRA Paper No. 61354, posted 18 Jan 2015 07:35 UTC 


\title{
Modeling Path Dependent Counterparty Credit Risk
}

\author{
Richard Zhou ${ }^{1}$ \\ Wells Fargo Bank \\ January 8, 2015 \\ Version 1.0 \\ Summary
}

\begin{abstract}
Path dependent counterparty credit risk exposure modeling poses challenges. In this paper, we discuss practical models for consistent and accurate estimation of counterparty credit exposure involving path-dependent derivatives. We derive analytical formulas for standalone expected exposure (EE), potential future exposure (PFE) and unilateral CVA for swap, swaption and barrier option. These formulas are of practical importance to financial institutions that use standalone exposure profiles, as well as to facilitate model validation and benchmarking.
\end{abstract}

\section{Introduction}

Modeling counterparty credit risk exposure involving path-dependent instruments poses significant challenges. The main issue is that, at the valuation date, the actual type of a pathdependent instrument at future times is unknown at the valuation date. This is referred to as instrument aging. This kind of aging differs from the shortening of the instrument's time-tomaturity because time-to-maturity change does not alter instrument type.

Broadly speaking, there are two kinds of path-dependency. The first kind is what can be termed as automatic exercise, or European exercise, where an instrument either terminates or becomes an instrument of a different type if some predetermined condition is met during the life of the option. Under automatic exercise, no decision is required and exercise is automatic. Examples of automatic exercise include barrier options and physically settled European swaptions. The second kind can be loosely called optimal exercise, or American exercise, where the exercise decision is based on the principle of payoff maximization. Examples include American and Bermudan style options. Modeling American exercise is much more involved than modeling European exercise. In this paper, we focus on the automatic exercise.

Lomibao and Zhu (2006) proposed a conditional valuation method for the type of path-dependent options whose future values can be expressed as either a sum or a product of two terms where one term depends only on the path history and the other term is the future mark-to-market value of the option and is independent of the history. The model was presented based on the Direct-Jump to Simulation (DJS) scenario generation approach.

With DJS, the scenario at time $t$ is generated independently whereas with the pathwise approach an entire path is simulated. While the two approaches theoretically generate the same distribution, the pathwise approach is more accurate for path-dependent derivatives as it can take into account the path information.

\footnotetext{
${ }^{1}$ Email: rzhou50@gmail.com. The opinions in this paper are the author's own and are not in any way related to his employer. The author is solely responsible for the contents. All errors are the author's own.
} 
A more fundamental issue with the method is the inconsistency between European swaption exercise probability estimation and the underlying swap valuation which we will discuss in section 4.

In this paper, we present an alternative model for the estimation of the swaption exercise probability. The model is based on a one-factor short rate model. Since a single short rate process generates risk scenario for both swaption and swap, swaption exercise probability is consistent with the swap valuation.

Using barrier option as prototype, we extend the approach of Lomibao and Zhu (2006) to pathwise scenario generation, and show that taking into consideration of path information can significantly increase accuracy without significant overhead.

We also provide analytical EE and PFE formulas for swap, European swaption and barrier option. While practical portfolio exposure calculation most likely requires Monte Carlo simulation, standalone exposures on single instrument is still important. First, it is useful to compare standalone exposure with marginal or incremental exposure for a trade. Second, analytical exposure formulas are valuable for counterparty credit risk model benchmarking and validation.

The rest of the paper is organized as follows. Section 2 outlines exposure and CVA definitions and formulations. Section 3 describes an analytic interest rate swap exposure model in which the accrued interest is stochastic. Building on the results of section 3, section 4 deals with the European swaption exposures where we present a model for estimating the swaption exercise probability that is consistent with the underlying swap valuation. We derive analytic formulas for exposure calculation. In section 5, we discuss an accurate method for barrier option exposure as well as analytic exposure formulas. Section 6 concludes the paper. Details of derivation are provided in the appendixes.

\section{Exposure Definition}

Throughout this paper, we shall use superscripts $Q$ to denote the pricing measure, and $P$ to symbolize the scenario generation measure. Since pricing is usually under the risk-neutral (RN) measure, $Q$ generally refers to the RN measure. The scenario measure $P$ may be either real-world (RW) or RN depending on the context. For instance, trading book CVA requires RN scenarios and hence $P$ is also RN, whereas PFE and EE for capital purpose generally require RW scenarios and hence $P$ is RW. ${ }^{2}$

Consider a counterparty portfolio with cashflow $C F\left(T_{i}\right)$ on dates $T_{i} ; i=1, \cdots, N$. The portfolio mark-to-market value at future time $\mathrm{t}$ is

$$
V(t)=E^{Q}\left\{\sum_{i \geq j(t)} D^{Q}\left(t, T_{i}\right) C F\left(T_{i}\right) \mid \mathcal{F}_{t}^{P}\right\}
$$

where $j(t)=\min \left\{i \mid T_{i} \geq t ; i=1, \cdots, N\right\}$ is the next cashflow date after $t$. Note that $j\left(T_{k}\right)=T_{k}$ and $C F\left(T_{k}\right)$ is included in the current exposure (CE), because cashflow on or after counterparty default would normally be part of the default settlement. For example, net accrued interest in an interest rate swap is included in the $\mathrm{CE}$ on the payment date. $\mathcal{F}_{t}^{P}$ is the augmented $P$-measure filtration generated by the scenario model, meaning that $\mathcal{F}_{t}^{P}$ contains the information up to $t$ along a specific path. Alternatively, we can think of $\mathcal{F}_{t}^{P}$ as path identification. $D^{Q}(t, T)=e^{-\int_{t}^{T} r^{Q}(s) d s}$ is the stochastic discount factor under the $Q$-measure and differs from the zero-coupon bond (ZCB) price defined as

\footnotetext{
${ }^{2}$ The book by Brigo et al (2013) provides a comprehensive description of exposure definitions.
} 


$$
B(t, T)=B^{Q}(t, T)=E^{Q}\left\{D^{Q}(t, T) \mid \mathcal{F}_{t}^{P}\right\}
$$

In a scenario/revaluation model framework, an important issue is the consistency between scenario and pricing. In such a framework, the pricing is conditioned on the scenario path $\mathcal{F}_{t}^{P}$. Ideally, $B(t, T)$ should match the scenario interest rate curves if possible.

When using a term structure model (eg, LMM and HJM model) to generate interest rate scenario, by construct, the scenario filtration $\mathcal{F}_{t}^{P}$ contains the whole simulated yield curve which can be used as the initial yield curve for arbitrage pricing model. As a result, $B(t, T)$ matches exactly the scenario interest rate curve when conditioned on $\mathcal{F}_{t}^{P}$, provided that the pricing model takes the initial curve as input. Take, for example, the Hull-White model (Hull and White 1990) as the pricing model. The mean-reversion speed and the volatility are calibrated at time 0 . The reversion level can then be used to match the scenario yield curve at time t.

If the interest rate scenarios are generated by a short rate model, then the short rate $r_{t}^{P}$, not the yield curve, is the risk scenario. The short rate $r_{t}^{P}$ says nothing about the scenario yield curve at time $t$. In this case, one strategy is to calibrate the pricing model to the initial market condition, and then use the calibrated pricing model to generate the yield curve $B(t, T)$ defined in (2.2). This is the strategy we assume to derive the analytic exposure formulas later in this paper.

The current exposure at time $\mathrm{t}$, which is the maximum amount the bank is at risk should the counterparty default immediately, is defined as $C E(t)=V(t)^{+}=\operatorname{Max}(V(t), 0)$. The expected exposure (EE) and negative expected exposure (NEE) are defined by

$$
E E(t)=E^{P}\left\{V(t)^{+} \mid \mathcal{F}_{0}\right\}, N E E(t)=E^{P}\left\{[-V(t)]^{+} \mid \mathcal{F}_{0}\right\}=E E(t)-E^{P}\left\{V(t) \mid \mathcal{F}_{0}\right\}
$$

EE is the maximum credit loss to the bank should the counterparty default, and NEE is the maximum benefit to the bank should the bank default. NEE is required for DVA calculation. It is important to note that, as indicated by (2.1) and (2.3), the portfolio value $V(t)$ is evaluated under the measure $Q$ while EE and NEE are evaluated under the measure $P$.

$V(t)$ is conditioned on augmented scenario filtration $\mathcal{F}_{t}^{P}$, and hence is dependent on a specific scenario path, or is pathwise. $E E(t)$ is the average value of $V(t)^{+}$over all such scenario paths conditional on the information at time 0 . As such, $E E(t)$ is a deterministic function of time. For capital purpose, $P$ is typically $\mathrm{RW}^{3}$, while for trading book CVA, $P$ is risk-neutral because CVA is a market price of the counterparty credit risk and is often hedged by the bank. EE in the context of CVA is easier than EE for capital purpose in the sense CVA requires only risk-neutral measure whereas risk calculation requires two measures. However, CVA is generally hedged so sensitivity to market risk factors are also important.

The PFE for confidence level $\alpha$ is defined as

$$
\begin{aligned}
P F E_{\alpha}(t) & =\operatorname{Max}\left\{x \mid P^{P}\left[V(t)^{+} \geq x \mid \mathcal{F}_{0}\right] \geq 1-\alpha\right\} \\
& =\left(\operatorname{Max}\left\{x \mid P^{P}\left[V(t) \geq x \mid \mathcal{F}_{0}\right] \geq 1-\alpha\right\}\right)^{+}
\end{aligned}
$$

\footnotetext{
${ }^{3}$ Although the regulators do not require scenario models to be RW, the regulatory mandate that scenario models must pass backtesting suggests that that should be the case.
} 
(2.4) shows that $P F E_{\alpha}(t)$ is the zero-floored $\alpha$-percentile of the portfolio value distribution at $t$ seen at time 0 . The second expression in (2.4) indicates that PFE is similar to VaR where the similarity stems from the fact that both are quantile measures.

Remark 2.1: Contrary to some belief, $P F E_{\alpha}(t)$ can actually be less than $E E(t)$, because $P F E_{\alpha}(t)$ dose not account for the distribution tail beyond the confidence level $\alpha$, whereas $E E(t)$ accounts for the entire distribution. For example, consider a forward contract on a stock, the EE and PFE are

$$
E E(t)=S_{0} \Phi\left(d_{+}\right)-K \Phi\left(d_{-}\right)>0, P F E_{\alpha}(t)=\left(S_{0} e^{-0.5 \sigma^{2} t+\sigma \sqrt{t} \epsilon_{\alpha}}-K\right)^{+}, d_{ \pm}=\frac{\ln \left(S_{0} / K\right)}{\sigma \sqrt{t}} \pm \frac{1}{2} \sigma \sqrt{t}
$$

where $\epsilon_{\alpha}=\Phi^{-1}(\alpha)$. Suppose $T<\left(\frac{\epsilon_{\alpha}}{\sigma}\right)^{2}$, then if $S_{0}<K / \max _{t \leq T} e^{-0.5 \sigma^{2} t+\sigma \sqrt{t} \epsilon_{\alpha}}=e^{-0.5 \sigma^{2} T+\sigma \sqrt{T} \epsilon_{\alpha}}$, we have $P F E_{\alpha}(t)=0$, ie, $P F E_{\alpha}(t)<E E(t)$ for all $t \leq T$.

CVA is the price adjustment due to the possibility of counterparty default causing financial loses to the non-defaulting party. Since it is a price adjustment, CVA should be evaluated under the $Q$ measure. A comprehensive CVA model would account for such effects as first-to-default, wrongway risk, collateralization, etc. The first-to-default effect has been shown to be significant (Brigo et al 2011), and is also shown to be non-negligible in the presence of a credit rating trigger (Zhou 2013). Neglecting the first-to-default in CVA is equivalent to assuming the bank is default-free. Wrong-way risk can be dominant for certain types of portfolio. Nevertheless, we do not consider these effects in this paper. Under the simplifying assumptions outlined above, the unilateral CVA at time $t$ is defined as

$$
\begin{aligned}
\operatorname{UCVA}(t) & =1\left(\tau^{P}>t\right) E^{Q}\left\{\delta_{C} D\left(t, \tau^{Q}\right) V\left(\tau^{Q}\right)^{+} \mid \mathcal{F}_{t}^{P}\right\} \\
& =1\left(\tau^{P}>t\right) \int_{t}^{T} E^{Q}\left\{\delta_{C} D(t, s) V(s)^{+} \mid \mathcal{F}_{t}^{P}\right\} d F_{t}^{Q}(s)
\end{aligned}
$$

where $\tau^{P}$ is the default time of the counterparty under the $P$-measure, $T$ is the final maturity of the counterparty portfolio, $\delta_{C}$ is the loss-given-default (LGD). $F_{t}^{Q}(s)$ is the $Q$-measure cumulative default probability of the counterparty conditional on surviving to t. $1\left(\tau^{P}>t\right)$ implies that CVA vanishes after the counterparty default. $U C V A(t)$ depends on the simulated "market" condition $\mathcal{F}_{t}^{P}$.

The traditional trading book CVA is equal to $\operatorname{UCVA}(0)$,

$$
U C V A=\int_{0}^{T} E^{Q}\left\{\delta_{C} D(0, s) V(s)^{+} \mid \mathcal{F}_{0}\right\} d F_{0}^{Q}(s)
$$

When cashflows are all positive, ${ }^{4}$ then $E^{Q}\left\{D(0, s) V(s)^{+} \mid \mathcal{F}_{0}\right\}=E^{Q}\left\{\sum_{i \geq j(s)} D\left(0, T_{i}\right) C F\left(T_{i}\right) \mid \mathcal{F}_{0}\right\}$, and (2.6) can be simplified as

$$
U C V A=\delta_{C} \sum_{k=1}^{N}\left(\sum_{i \geq k} E^{Q}\left\{D\left(0, T_{i}\right) C F\left(T_{i}\right) \mid \mathcal{F}_{0}\right\}\right)\left(F_{0}^{Q}\left(T_{k}\right)-F_{0}^{Q}\left(T_{k-1}\right)\right)
$$

where $F_{0}^{Q}(t)$ is the risk-neutral counterparty default probability which can be inferred from either the market credit spread if the counterparty has liquid CDS trades or rating-based generic credit spread otherwise. Since the counterparty is alive today, we have $F_{0}^{Q}(0)=0$.

${ }^{4}(2.7)$ is convenient for option exposure modeling as cashflows on options are generally positive. 
In the above discussion, we have used $P$-measure and $Q$-measure to symbolize the Monte Caro scenario generation and instrument pricing. But we did not impose any relationship between the two measures. It is desirable to treat the two measures as distinct because the scenario model may be calibrated to the historical volatility while the pricing model is calibrated to the market implied volatility.

\section{Interest Rate Swaps}

Fixed-for-floating interest rate swaps and European swaptions are the most widely traded OTC derivatives. Global banks have substantial swap books. Swaps are also convenient cases for counterparty exposure model validation due to its simplicity. While frequently used as an example in literature and often among the first instruments to be covered in a newly developed CCR system, there still appears to be a practical need for a detailed, rigorous exposition on the profile of swap value and exposure.

In this section, we discuss in detail swap valuation and exposure. We pay special attention to the jump in swap value across a payment date because it is the primary reason that swap exposure profile exhibit a sawtooth pattern.

Consider an interest rate swap exchanging the Libor for a fixed rate $\mathrm{K}$. The swap starts at $T_{0}=S_{0}$ and ends at $T_{N}=S_{M}$, with the floating rate payment dates $T_{1}<\cdots<T_{N}$ and the fixed rate payment dates $S_{1}<\cdots<S_{M}=T_{N}$. Assuming Libor fixing and discounting, ${ }^{5}$ the future mark-to-market swap value to the fixed rate payer is

$$
V_{S w a p}(t)=\left\{\begin{array}{lr}
B\left(t, T_{0}\right)-B\left(t, T_{N}\right)-K \sum_{m=1}^{M} B\left(t, S_{m}\right) \Delta S_{m-1}=V_{F w d S w a p}(t), & t \leq T_{0} \\
\frac{B\left(t, T_{\alpha(t)}\right)}{B\left(T_{\left.\alpha(t)-1, T_{\alpha(t)}\right)}\right.}-B\left(t, T_{N}\right)-K \sum_{m=\beta(t)}^{M} B\left(t, S_{m}\right) \Delta S_{m-1}=V_{\text {Tailswap }}(t), t>T_{0}
\end{array}\right.
$$

where $\Delta S_{m-1}=S_{m}-S_{m-1} \cdot \alpha(t)=\operatorname{Max}\left\{\operatorname{Min}\left\{k \mid T_{k} \geq t\right\}, 0\right\}$ and $\beta(t)=\operatorname{Max}\left\{\operatorname{Min}\left\{m \mid S_{m} \geq t\right\}, 0\right\}$ are the next fixed and floating rate payment dates, respectively. $\alpha(t)$ and $\beta(t)$ are continuous from the left in order for the accrued interests to be included in the exposure. It is important to point out that $\lim _{t \downarrow T_{0}} V_{\text {Tailswap }}(t)=V_{F w d S w a p}\left(T_{0}\right)$. Since $t$ is a simulation date, the zero-coupon bond $B(t, T)$ is known for all T. But $B\left(T_{\alpha(t)-1}, T_{\alpha(t)}\right)$ may be unknown because $T_{\alpha(t)-1}$ is generally not a simulation date. As a result, $B\left(T_{\alpha(t)-1}, T_{\alpha(t)}\right)$ is either approximated or modeled. Stein and Lee (2010) assumed that $B\left(T_{\alpha(t)-1}, T_{\alpha(t)}\right)$ is equal to the time 0 forward value, which is equivalent to assuming the spot Libor at $T_{\alpha(t)-1}$ to be its time 0 forward value. We will discuss a model for $B\left(T_{\alpha(t)-1}, T_{\alpha(t)}\right)$ in Appendix B.

In (3.1), $V_{\text {FwdSwap }}(t)$ and $V_{\text {Tailswap }}(t)$ are values of a forward swap and a tail (seasoned) swap, respectively. The tail swap can be expressed a forward swap starting at $T_{\alpha(t)}$ plus a net interest accrued to $T_{\alpha(t)}$. To fix the idea, we assume that any fixed date is also a floating, $\left\{S_{1}, \cdots, S_{M}\right\} \subseteq$ $\left\{T_{1}, \cdots, T_{N}\right\}$ implying $S_{\beta(t)} \geq T_{\alpha(t)}$. Thus

\footnotetext{
${ }^{5}$ Collateralized swaps are discounted at the collateral rate not Libor which requires multi-curve modeling.
} 


$$
V_{\text {Tailswap }}(t)=\left\{\begin{array}{c}
1-B\left(t, T_{N}\right)-K\left[\sum_{m=\beta(t)+1}^{M} B\left(t, S_{m}\right) \Delta S_{m-1}+B\left(t, S_{\beta(t)}\right)\left(S_{\beta(t)}-t\right)\right] \\
+\frac{B\left(t, T_{\alpha(t)}\right)}{B\left(T_{\alpha(t)-1}, T_{\alpha(t)}\right)}-1-K B\left(t, S_{\beta(t)}\right)\left(t-S_{\beta(t)-1}\right)
\end{array}\right\}
$$

The top line in (3.2) is the value of the spot $T_{N}$-maturity swap with the floating payment dates $T_{\alpha(t)}, T_{\alpha(t)+1}, \cdots, T_{N}$ and the fixed rate payment dates $S_{\beta(t)}, S_{\beta(t)+1}, \cdots, S_{M}$. The bottom line represents the net accrued interest from the last floating and fixed dates to $t$ which we will discuss below.

Noticing that $L\left(T_{n-1}, T_{n-1}\right)=\left(B\left(T_{n-1}, T_{n}\right)^{-1}-1\right) / \Delta T_{n-1}$ is the spot Libor for period $\left(T_{n-1}, T_{n}\right)$ which is known when conditioned on $\mathcal{F}_{t}^{P}$, the bottom line in (3.2) can be rewritten as

$$
N A I(t)=\left\{\begin{array}{c}
L\left(T_{\alpha(t)-1}, T_{\alpha(t)-1}\right) \Delta T_{\alpha(t)-1} B\left(t, T_{\alpha(t)}\right)-\left[1-B\left(t, T_{\alpha(t)}\right)\right] \\
-K B\left(t, S_{\beta(t)}\right)\left(t-S_{\beta(t)-1}\right)
\end{array}\right\}
$$

(3.3) shows that the net accrued interest at time $t$ is equal to the floating coupon of the original swap for $\left(T_{\alpha(t)-1}, T_{\alpha(t)}\right)$ adjusted for the portion that is included in the new swap minus the fixed coupon accrued from the last fixed payment date $S_{\beta(t)-1}$. It is easy to see that if $t=T_{\alpha(t)}=S_{\beta(t)}$, then $N A I(t)=L\left(T_{\alpha(t)-1}, T_{\alpha(t)-1}\right) \Delta T_{\alpha(t)-1}-K B\left(t, S_{\beta(t)}\right) \Delta S_{\beta(t)-1}$, the full net coupon on the original swap, whereas if $t=T_{\alpha(t)}+=S_{\beta(t)}+$, then $N A I(t)=0$. In other words, immediately before a payment date, NAI is the full accrued coupon, whereas immediately after a payment, the accrued interest is zero.

Put another way, $V_{\text {Swap }}(t)$ is continuous everywhere except at the coupon payment dates $T_{n}$ and $S_{m}$. Let $T$ be either a floating date $T_{n}$ or a fixed date $S_{m}$, then the swap value is left continuous, $V_{S w a p}(T)=\lim _{t \uparrow T} V_{S w a p}(t)$, and right limited, $\lim _{t \downarrow T} V_{S w a p}(t)=V_{S w a p}(T+)$. This is a consequence of how $\alpha(t)$ and $\beta(t)$ are defined. It is easy to verify that $N A I(T+)=0$ if $T$ is a payment date for both fixed and floating legs.

The change in swap value across a floating rate payment date $T_{n}$ is

$$
V_{\text {Swap }}\left(T_{n}+\right)-V_{\text {Swap }}\left(T_{n}\right)=K \Delta S_{\beta\left(T_{n}\right)-1} 1\left(S_{\beta\left(T_{n}\right)}=T_{n}\right)-L\left(T_{n-1}, T_{n-1}\right) \Delta T_{n-1}
$$

(3.4) implies that the pathwise value of a payer swap decreases by $L\left(T_{n-1}, T_{n-1}\right) \Delta T_{n-1}$ after a float payment (one less future receipt), and increases by $K \Delta S_{\beta\left(T_{n}\right)-1}$ after a fixed payment (one less future payment). The jump magnitude depends on the difference between the fixed and floating rates and their respective accrued periods. This jump is the reason why swap exposure profile typically exhibits a sawtooth pattern.

The change in the pathwise swap value over the time interval $\left(T_{n-1}, T_{n}\right)$ is

$$
V_{S w a p}\left(T_{n}\right)-V_{S w a p}\left(T_{n-1}+\right)=\frac{1}{B\left(T_{n-1}, T_{n}\right)}-1-\Delta B\left(T_{n-1}, T_{N}\right)-K \sum_{m=\beta\left(T_{n}\right)}^{M} \Delta B\left(T_{n-1}, S_{m}\right) \Delta S_{m-1}
$$


where $\Delta B\left(T_{n-1}, U\right)=B\left(T_{n}, U\right)-B\left(T_{n-1}, U\right)$. (3.5) is due entirely to diffusion effect and passage of time. Its effect on the exposure profile is gradual and smooth.

Define the forward swap rate and annuity for a $T_{N}$-maturity swap that starts at $s \geq t$ with fixed payment dates $\left\{S_{\beta(s)}, \cdots, S_{M}\right\}$ and floating payment dates $\left\{T_{\alpha(s)}, \cdots, T_{N}\right\}$

$$
R(t ; s)=\frac{B(t, s)-B\left(t, T_{N}\right)}{A(t ; s)}, A(t ; s)=\sum_{m=\beta(s)+1}^{M} B\left(t, S_{m}\right) \Delta S_{m-1}+B\left(t, S_{\beta(s)}\right)\left(S_{\beta(s)}-s\right)
$$

The forward swap rate for the original swap is $R\left(t ; T_{0}\right)$, and the swap rate for the first line of (3.2) is $R(t ; t)$. With (3.6), we can recast the swap value (3.1) into

$$
V_{\text {Swap }}(t)= \begin{cases}V_{\text {FwdSwap }}(t)=\left(R\left(t ; T_{0}\right)-K\right) A\left(t ; T_{0}\right), & t \leq T_{0} \\ V_{\text {Tailswap }}(t)=(R(t ; t)-K) A(t ; t)+N A I(t), & t>T_{0}\end{cases}
$$

By virtue of (2.3) and (3.1), the standalone EE for the payer swap is

$$
E E_{\text {Swap }}(t)=E^{P}\left\{\left(\begin{array}{l}
V_{\text {FwdSwap }}(t)^{+} \\
V_{\text {Tailswap }}(t)^{+}
\end{array}\right) \mid \mathcal{F}_{0}\right\}=\left\{\begin{array}{c}
E^{P}\left\{\left(R\left(t ; T_{0}\right)-K\right)^{+} A\left(t ; T_{0}\right) \mid \mathcal{F}_{0}\right\}, t \leq T_{0} \\
E^{P}\left\{(R(t ; t)-\bar{K}(t))^{+} A(t ; t) \mid \mathcal{F}_{0}\right\}, t>T_{0}
\end{array}\right.
$$

where

$$
\bar{K}(t)=K-\frac{N A I(t)}{A(t ; t)}=K+\frac{1+K B\left(t, S_{\beta(t)}\right)\left(t-S_{\beta(t)-1}\right)}{A(t ; t)}-\frac{\left[L\left(T_{\alpha(t)-1}, T_{\alpha(t)-1}\right) \Delta T_{\alpha(t)-1}+1\right] \mathrm{B}\left(t, T_{\alpha(t)}\right)}{A(t ; t)}
$$

A positive (negative) $N A I$ reduces (increases) $\bar{K}(t)$ and hence increases (decreases) the exposure of the tail swap. It is possible for $\bar{K}(t)$ to be negative when $K$ is low or $L\left(T_{\alpha(t)-1}, T_{\alpha(t)-1}\right)$ is high.

It is important to point out that although the top line of $(3.8), E^{P}\left\{\left(R\left(t ; T_{0}\right)-K\right)^{+} A\left(t ; T_{0}\right) \mid \mathcal{F}_{0}\right\}$, resembles a European swaption, but it is not. The primary reason is that it is the undiscounted expectation. In addition, if the two measures are different, $R\left(t ; T_{0}\right)$ is not a martingale under a measure equivalent to the $P$-measure.

In Appendix A, we will discuss analytic formulas for valuation of (3.8) based on the one-factor Hull-White model, where (A.7) is for $E^{P}\left\{V_{\text {Fwdswap }}(t)^{+} \mid \mathcal{F}_{0}\right\}$ and (A.24) for $E^{P}\left\{V_{\text {Tailswap }}(t)^{+} \mid \mathcal{F}_{0}\right\}$. These formulas are valuable for validation, benchmarking and impact assessment. In the remainder of this section, we discuss casting $E^{P}\left\{V_{\text {Tailswap }}(t)^{+} \mid \mathcal{F}_{0}\right\}$ as a forward starting swaption that may facilitate calculation under the Libor Market Model.

Brace and Womersley (2000) showed that $B(t, T) / A(t ; t)$ is a low variance martingale and can be approximated by its time 0 value. Thus, the effective strike can be approximated as

$$
\bar{K}(t) \cong K\left[1+\frac{B\left(0, S_{\beta(t)}\right)\left(t-S_{\beta(t)-1}\right)}{A(0 ; t)}\right]+\frac{1-B\left(0, T_{\alpha(t)}\right)}{A(0 ; t)}-\frac{B\left(0, T_{\alpha(t)}\right) \Delta T_{\alpha(t)-1}}{A(0 ; t)} L\left(T_{\alpha(t)-1}, T_{\alpha(t)-1}\right)
$$


(3.8) and (3.10) suggest that $E^{P}\left\{V_{\text {Tailswap }}(t)^{+} \mid \mathcal{F}_{0}\right\}$ could be mathematically considered as a forward starting swaption with the strike being a linear function of the Libor resetting at $T_{\alpha(t)-1}$. Evaluation of (3.8) with stochastic strike given by (3.10) should take into account the correlation between the spot swap rate $R(t ; t)$ and the spot Libor $L\left(T_{\alpha(t)-1}, T_{\alpha(t)-1}\right)$, as well as the forward swaption volatility skew. Stein et al (2010) assumed $L\left(T_{\alpha(t)-1}, T_{\alpha(t)-1}\right) \cong L\left(0, T_{\alpha(t)-1}\right)$ to assess the difference in CVA between the forward swap (no NAI $(t)$ in (3.8)) and the tail swap. However, the impact of this assumption on EE and PFE was not addressed, which can be potentially substantial for long-dated swaps. This impact can be accessed using the method in Appendices A and B.

\section{Physically Settled European Swaptions}

Unlike swaption pricing that ends at the swaption expiration, the exposure of a physically settled swaption can persist long after the option has expired. While what happens after the swaption expiry date is irrelevant to the option pricing, it has major impact on the exposure. To make the matter even more complicated, whether the swaption would be exercised and hence becoming the swap is not known a priori, making European swapition exposure path-dependent, even though the option itself is not. As such, a key element of modeling physically settled European swaption exposure is estimating the probability of swaption exercise.

We consider a swap-settled European payer swapion that gives the option holder the right to enter into the swap paying fixed rate $K$ where the swap terms are specified in the previous section. The future value of the European swaption is

$$
V_{\text {Swpn }}(t)=\left\{\begin{array}{cr}
E^{Q}\left\{D^{Q}\left(t, T_{0}\right) V_{F w d S w a p}\left(T_{0}\right)^{+} \mid \mathcal{F}_{t}^{P}\right\}=V_{\text {Swpn }}^{m t m}(t), & t \leq T_{0} \\
V_{\text {Tailswap }}(t) 1\left(V_{\text {FwdSwap }}\left(T_{0}\right)>0\right), & t>T_{0}
\end{array}\right.
$$

where $1(x)$ is the indicator function. The bottom line of (4.1) means that if the swap value at $T_{0}$ is not positive, the option is not exercised and there is no exposure afterwards. On the other hand, if the swap value at $T_{0}$ is positive, the option turns into a tail swap and the exposure persists until the swap maturity $T_{N}$.

The standard front office model for European swaption is the Black-Scholes model

$$
V_{S w p n}^{m t m}(t)=A\left(t ; T_{0}\right) C_{B S}\left(R\left(t ; T_{0}\right), K, 0, T_{0}-t, \sigma_{i m p}\right)
$$

where

$$
C_{B S}(S, K, r, \tau, \sigma)=S \Phi\left(d_{+}\left(\frac{S}{K}, r, \tau, \sigma\right)\right)-K e^{-r \tau} \Phi\left(d_{-}\left(\frac{S}{K}, r, \tau, \sigma\right)\right)
$$

with

$$
d_{ \pm}(x, r, \tau, \sigma)=\frac{\ln (x)}{\sigma \sqrt{\tau}}+\left(\frac{r}{\sigma} \pm \frac{1}{2} \sigma\right) \sqrt{\tau}
$$


It is important to note that $V_{\text {Tailswap }}(t)$ and $V_{F w d S w a p}\left(T_{0}\right)$ are evaluated on the same scenario path. When $T_{0}$ is a simulation node, $V_{F w d S w a p}\left(T_{0}\right)$ is available from the simulation and thus computation of the exercise indicator $1\left(V_{F w d S w a p}\left(T_{0}\right)>0\right)$ poses no problem. On the one hand, a practical portfolio typically contains many European swaptions with differing expiry dates. On the other hand, a CCR system normally has a fixed simulation date grid. As a result, it is likely that most swaption maturities are not on the simulation date grid. Consequently, the pathwise exercise indicator $1\left(V_{F w d S w a p}\left(T_{0}\right)>0\right)$ must be estimated. Since this indicator is a pathwise variable, it requires to be computed only once per scenario path.

Let the simulation date grid be denoted by $H=\left\{h_{1}, h_{2}, \cdots, h_{L}\right\}$ and assume $h_{k-1}<T_{0} \leq h_{k}$, the indicator $1\left(V_{\text {FwdSwap }}\left(T_{0}\right)>0\right)$ under the $P$-measure may be directly sampled conditional on $\mathcal{F}_{h_{k}}^{P}$. Given that $\mathcal{F}_{h_{k-1}}^{P} \subset \mathcal{F}_{h_{k}}^{P}$, this indicator takes value 1 with probability $P^{P}\left(V_{F w d s w a p}\left(T_{0}\right)>0 \mid \mathcal{F}_{h_{k}}^{P}\right)$. Thus, we have $1\left(V_{F w d S w a p}\left(T_{0}\right)>0\right)=P^{P}\left(V_{F w d S w a p}\left(T_{0}\right)>0 \mid \mathcal{F}_{T_{0}}^{P}\right)$, and the swaption value (4.1) can be rewritten as

$$
V_{\text {Swpn }}(t)=\left\{\begin{array}{l}
V_{\text {Swpn }}^{m t m}(t)=E^{Q}\left[D^{Q}\left(t, T_{0}\right) V_{F w d S w a p}\left(T_{0}\right)^{+} \mid \mathcal{F}_{t}^{P}\right], t \leq T_{0} \\
V_{\text {Tailswap }}(t) P^{P}\left(V_{F w d S w a p}\left(T_{0}\right)>0 \mid \mathcal{F}_{h_{k}}^{P}\right), \quad t>T_{0}
\end{array}\right.
$$

Direct simulation is inefficient in large scale computation, a practical approach is to estimate $P^{P}\left(V_{F w d S w a p}\left(T_{0}\right)>0 \mid \mathcal{F}_{h_{k}}^{P}\right)$. Since $1\left(V_{F w d S w a p}\left(T_{0}\right)>0\right)=1\left(R\left(T_{0} ; T_{0}\right)>K\right)$, then $P^{P}\left(V_{F w d S w a p}\left(T_{0}\right)>\right.$ $0 / \mathcal{F} h k P=P P R T 0 ; T 0>K \mid \mathcal{F} h k P$, the conditional exercise probability may be modeled using either the swap value or the swap rate. In some case, swap value may be more convenient as we will show below.

In an influential paper, Lomibao \& Zhu (2006) proposed a conditional valuation approach for modeling EE of path-dependent instruments. Their method is intended for the type of path dependent derivatives where the future value of the option can be expressed as either a product (cf. (4.4)) or a sum of future MTM value and a term that depends only on the path history. ${ }^{6}$ Here, future MTM value is the price at a future date of a newly issued instrument of the same type as the original instrument, and hence is independent of the path. The path-dependent term is irrelevant to the pricing, but is essential to exposure calculation. The central theme of the method is to use the Brownian bridge theory to estimate the path-dependenct term. When applied to estimate the swaption exercise probability $P^{P}\left(R\left(T_{0} ; T_{0}\right)>K \mid \mathcal{F}_{h_{k}}^{P}\right)$, it is assumed that the all $T_{N^{-}}$ maturity co-terminal swap rates follow a common lognormal process. This enables to link the swap rates of two distinct co-terminal swaps by $R\left(h_{k} ; h_{k}\right)=R\left(T_{0} ; T_{0}\right) e^{\left(\mu-0.5 \sigma^{2}\right)\left(h_{k}-T_{0}\right)+\sigma z \sqrt{h_{k}-T_{0}}}$. If $R\left(h_{k} ; h_{k}\right)$ is known from the simulation, $R\left(T_{0} ; T_{0}\right)$ can be estimated using a Brownian bridge between 0 and the simulation date $h_{k}$.

The method is elegant, simple and efficient. However, for swap-settled European swaption, it lacks theoretical justification as it is inconsistent with the swap pricing. First, $R\left(t ; T_{0}\right)$ is the swap rate of an $N$ period swap underlying the swaption, and $R\left(t ; h_{k}\right)$ is the swap rate for a $\left(N-\alpha\left(h_{k}\right)\right)$

\footnotetext{
${ }^{6}$ This excludes the American and Bermudan style options for which such separation is impossible.
} 
period swap. (3.6) indicates that $R\left(t ; T_{0}\right)$ and $R\left(t ; h_{k}\right)$ generally cannot follow the same lognormal process. To illustrate the point, consider a 6-month swaption into a one-year swap receiving 6 month Libor flat with semi-annual payment. Suppose the simulation time nodes are 6 months apart then we get $h_{k}=T_{1}=$ one year. $R_{0}(t)$ is the one-year swap rate which is a weighted average of two adjacent forward 6-month Libor spanning the one-year swap, while $R_{1}(t)$ is the 6-month forward Libor for the last 6-month of the one-year swap. Clearly, the one year swap rate and the 6-month Libor generally do not follow the same process. Second, since $R\left(t ; T_{0}\right)$ and $R\left(t ; h_{k}\right)$ represent swaps of different tenors, they should have different volatilities, irrespective of measure. This raises the question of which volatility $\sigma$ to use for the Brownian bridge model. Third, these issues are likely exacerbated by the fact that a typical counterparty portfolio contains many swaps and swaptions.

The above analysis suggests that estimation of $P^{P}\left(V_{F w d S w a p}\left(T_{0}\right)>0 \mid \mathcal{F}_{h_{k}}^{P}\right)$ should be consistent with the swap pricing, which in turn suggests that a method may be based on the scenario and pricing models. In the following, we discuss a simple, practical method for consistent calculation of the exercise probability.

Suppose that the interest rate scenarios are generated by the short rate model

$$
d f\left(r_{t}^{P}\right)=\kappa^{P}\left(\theta^{P}-f\left(r_{t}^{P}\right)\right) d t+\sigma^{P} d w_{t}^{P}
$$

where $f(x)$ is an increasing function. If $(x)=x$, (4.5) represents the Vasicek model, while $f(x)=\ln (x)$ is the exponential Vasicek model.

Suppose that we have another (risk-neutral) short rate model to calculate the zero bond price (2.2) which we denote by $B\left(t, T, r_{t}^{P}\right)$. The actual bond pricing formula is not important to our exposition except that $B\left(t, T, r_{t}^{P}\right)$ is a decreasing function of $r_{t}^{P}$ which is satisfied by any sensible interest rate model. $^{7}$

Since the swap value at the swaption expiry date

$$
V_{\text {FwdSwap }}\left(T_{0}, r_{T_{0}}^{P}\right)=1-B\left(T_{0}, T_{N}, r_{T_{0}}^{P}\right)-K \sum_{m=1}^{M} B\left(T_{0}, S_{m}, r_{T_{0}}^{P}\right) \Delta S_{m-1}
$$

is a strictly increasing function of $r_{T_{0}}^{P}$, there is an unique value $r_{c}$ such that $V_{S w a p}\left(T_{0}, r_{c}\right)=0$. This implies that $V_{\text {Swap }}\left(T_{0}, r_{T_{0}}^{P}\right)>0$ if and only if $r_{T_{0}}^{P}>r_{c}{ }^{8}$ In other words, the payer swaption is exercised iff $r_{T_{0}}^{P}>r_{c}$. The critical rate $r_{c}$ is unique for a given swap, and needs to be computed once.

Using the Brownian bridge technique, the swaption exercise probability is

$$
P^{P}\left(V_{\text {FwdSwap }}\left(T_{0}\right)>0 \mid \mathcal{F}_{h_{k}}^{P}\right)=P^{P}\left(f\left(r_{T_{0}}^{P}\right)>f\left(r_{c}\right) \mid f\left(r_{h_{k}}^{P}\right), f\left(r_{h_{k-1}}^{P}\right)\right)
$$

\footnotetext{
${ }^{7}$ For affine models, $B\left(t, T, r_{t}^{P}\right)=e^{A(t, T)-C(t, T) r_{t}^{P}}$ where $C(t, T)$ is a positive functions of $t$ and $T$.

${ }^{8}$ For receiver swap, $V_{F w d S w a p}\left(T_{0}, r_{T_{0}}^{P}\right)>0$ iff $r_{T_{0}}^{P}<r_{c}$.
} 


$$
=\Phi\left(\left\{f\left(r_{h_{k-1}}^{P}\right) p_{T_{0}}+f\left(r_{h_{k}}^{P}\right) q_{T_{0}}+\tilde{\mu}_{T_{0}}-f\left(r_{c}\right)\right\} / \sigma_{T_{0}}^{B B}\right)
$$

The derivation is provided in Appendix C. Here we summarize some important characteristics of (4.7) due to mean reversion

- The coefficient $p_{t}$ and $q_{t}$ are positive with $p_{t}+q_{t} \leq 1$ where the equality holds only at the bridge endpoints. Given $h_{k-1}$ and $h_{k}$, they depend only on the mean-reversion speed $\kappa^{P}$.

- $\tilde{\mu}_{t}=\theta^{P}\left(1-p_{t}-q_{t}\right)$.

- $\sigma_{t}^{B B}$ is proportional to $\sigma^{P}$ and $\sigma_{h_{k-1}}^{B B}=\sigma_{h_{k}}^{B B}=0$, but the variance $\left(\sigma_{t}^{B B}\right)^{2}$ is not a quadratic function of $\mathrm{t}$.

- The standard Brownian bridge results with constant drift (Shreve 2004) is recovered when $\kappa^{P} \rightarrow 0$.

Let $h_{k-1} \rightarrow T_{0}$, (4.7) shows that $P^{P}\left(V_{F w d S w a p}\left(T_{0}\right)>0 \mid \mathcal{F}_{h_{k}}^{P}\right)=1(0)$ if $f\left(r_{h_{k-1}}^{P}\right)>(<) f\left(r_{c}\right)$. The same holds for $h_{k} \rightarrow T_{0}$. These limiting cases indicate that (4.7) is consistent when $T_{0}$ is a simulation date in which case we know for sure whether there will be a swap after the expiry date. The probability of one or zero can only be attained at the two end points. Therefore, the swaption exposure post expiry date must be less than that of the underlying swap.

For the non-trivial case where $h_{k-1}<T_{0}<h_{k}$, (4.7) indicates that the higher are the values of $f\left(r_{h_{k-1}}^{P}\right)$ and/or $f\left(r_{h_{k}}^{P}\right)$, the greater is the exercise probability, and vice versa. The interest rate volatility is important to the exercise probability. Clearly, $P^{P}\left(V_{S w a p}\left(T_{0}\right)>0 \mid \mathcal{F}_{h_{k}}^{P}\right) \rightarrow 1 / 2$ when $\sigma^{P} \rightarrow \infty$, regardless of the values of $r_{h_{k-1}}^{P}, r_{h_{k}}^{P}$ and $r_{c}$. For large interest rate volatility, one should expect substantial difference in exposure between the swaption and the underlying swap. For small volatility, the exercise should be close to one if $f\left(r_{h_{k-1}}^{P}\right) p_{T_{0}}+f\left(r_{h_{k}}^{P}\right) q_{T_{0}}>f\left(r_{c}\right)-\tilde{\mu}_{T_{0}}$ and close to zero otherwise. These characteristics may be useful for model validation purpose.

Finally, the conditional exercise probability is independent of the interest rate drift which is an artifact of the Brownian bridge as the drift is implicit in the values at the two bridge endpoints.

Substituting (4.4) into (2.3), the EE of a standalone European swaption is

$$
E E_{\text {Swpn }}(t)=\left\{\begin{array}{cr}
E^{P}\left\{V_{\text {Swpn }}^{m t m}(t) \mid \mathcal{F}_{0}\right\}, & t \leq T_{0} \\
E^{P}\left[V_{\text {Tailswap }}(t)^{+} 1\left(V_{\text {FwdSwap }}\left(T_{0}\right)>0\right) \mid \mathcal{F}_{0}\right], & t>T_{0}
\end{array}\right.
$$

The swaption exposure prior to the expiration date may be calculated as follows. Suppose that $V_{S w p n}^{m t m}(t)$ is valued using (4.2), then

$$
E^{P}\left\{V_{S w p n}^{m t m}(t) \mid \mathcal{F}_{0}\right\}=E^{P}\left\{A\left(t ; T_{0}\right) C_{B S}\left(R\left(t ; T_{0}\right), K, T_{0}-t, \sigma_{i m p}\right) \mid \mathcal{F}_{0}\right\}
$$

where $C_{B S}\left(R\left(t ; T_{0}\right), K, T_{0}-t, \sigma_{\text {imp }}\right)$ is given by (4.3). The annuity $A\left(t ; T_{0}\right)$ and swap rate $R\left(t ; T_{0}\right)$ are defined in (3.6).

We now assume that pricing uses the Hull-White model, from (A.3) in appendix A, we have 


$$
\begin{aligned}
& A\left(r_{t}^{P}, t ; T_{0}\right)=\sum_{m=1}^{M} \Delta S_{m-1} e^{A^{Q}\left(t, S_{m}\right)-C^{Q}\left(t, S_{m}\right) r_{t}^{P}} \\
& R\left(r_{t}^{P}, t ; T_{0}\right)=\frac{e^{A^{Q}\left(t, T_{0}\right)-C^{Q}\left(t, T_{0}\right) r_{t-e^{A}}^{P}\left(t, T_{N}\right)-C^{Q}\left(t, T_{N}\right) r_{t}^{P}}}{A\left(t ; T_{0}\right)}
\end{aligned}
$$

This mean that, under the Hull-White model, $A\left(t ; T_{0}\right) C_{B S}\left(R\left(t ; T_{0}\right), K, T_{0}-t, \sigma_{\text {imp }}\right)$ is a function of the scenario short rate $r_{t}^{P}$. Furthermore, if the scenario model is given by (4.5), then

$$
x_{t}=f\left(r_{t}^{P}\right) \sim \Phi\left(f\left(r_{0}\right) e^{-k^{P} t}+\theta^{P}\left(1-e^{-k^{P} t}\right), \frac{\left(\sigma^{P}\right)^{2}}{2 k}\left(1-e^{-k^{P} t}\right)\right)=\Phi\left(\mu_{e}, \sigma_{e}^{2}\right)
$$

Hence,

$$
E^{P}\left\{V_{S w p n}^{m t m}(t) \mid \mathcal{F}_{0}\right\}=\frac{1}{\sqrt{2 \pi} \sigma_{e}} \int_{-\infty}^{\infty} A\left(f(x), t ; T_{0}\right) C_{B S}\left(R\left(f(x), t ; T_{0}\right), K, T_{0}-t, \sigma_{i m p}\right) e^{-\frac{1}{2}\left(\frac{x-\mu_{e}}{\sigma_{e}}\right)^{2}} d x
$$

Because $e^{-\frac{1}{2}\left(\frac{x-\mu_{e}}{\sigma_{e}}\right)^{2}}$ dominates as $|x| \rightarrow \infty$, the integral can be computed on a finite range. A semianalytic model for $E^{P}\left[V_{\text {Tailswap }}(t)^{+} 1\left(V_{\text {FwdSwap }}\left(T_{0}\right)>0\right) \mid \mathcal{F}_{0}\right]$ is given by (A.35) in Appendix A.

By virtue of (2.6) and (2.7), the standalone unilateral CVA of a swap-settled swaption is equal to

$$
U C V A_{S w p n}=\left\{\begin{array}{c}
\delta_{C} V_{S w p n}^{m t m}(0) F_{0}^{Q}\left(T_{0}\right)+ \\
\delta_{C} \int_{T_{0}}^{T_{N}} E^{Q}\left[D^{Q}(0, t) V_{\text {Tailswap }}(t)^{+} 1\left(V_{F w d S w a p}\left(T_{0}\right)>0\right) \mid \mathcal{F}_{0}\right] d F_{0}^{Q}(t)
\end{array}\right\}
$$

\section{Barrier Options}

A barrier option, including one-touch option, is path-dependent. If the underlying state variable reaches some pre-defined barrier level during the life of the barrier option, the option either ceases to exist (knocked out) or becomes a standard European option (knocked in). Modeling barrier option exposure is complicated because the future instrument type of a today's barrier option depends on what happens between now and then. A barrier option today may or may not be a barrier option tomorrow. In other words, barrier option exposure depends on the instrument aging. In the previous section, we discussed a model for the swap-settled European swaption exposure. European swaption exposure calculation requires monitoring the underlying swap value only at the option expiry date. In contrast, barrier option exposure requires continuous monitoring of the entire option life.

Consider a European up-and-out stock call option with rebate (UOR) with the terminal payoff

$$
g_{U O R}(T)=\phi\left(S_{T}-K\right)^{+} 1\left(\tilde{S}_{T}<H\right)+R 1\left(\tilde{S}_{T} \geq H\right)
$$

where $\tilde{S}_{t}=\max _{u \leq t} S_{u}$ is the running maximum of the stock price, $H$ is the barrier level, $K(<H)$ is the strike, and $\mathrm{R}$ is the rebate amount if the stock price $S_{t}$ crosses $H$ before the expiry date $T$. $\phi=1$ represents an UO call option while $\phi=0$ is a one-touch cash-or-nothing option that pays out $R$ at the expiry T if $S_{t}$ reaches $H$ during the option life and nothing otherwise. When $\phi=1$ and $R=H-K>0,(5.1)$ represents a European capped option where it is a call option but pays $H-K$ should the barrier be reached before expiration, capping the maximum payoff at $H-K$. 
The future MTM price of the UOR option is

$$
V_{U O R}^{m t m}(t)=E^{Q}\left\{D^{Q}(t, T)\left[\phi\left(S_{T}^{Q}-K\right)^{+} 1\left(\tilde{S}_{T}^{Q}<H\right)+R 1\left(\tilde{S}_{T}^{Q} \geq H\right)\right] \mid S_{t}^{P}\right\}
$$

It is the price of a new UOR option issued at time t when stock price is $S_{t}^{P}$. The first term is a standard up-and-out barrier option that pays nothing if the barrier is crossed during the life of the option. The second term is a one-touch option representing a contingent rebate. It is emphasized here that $V_{U O R}^{m t m}(t)$ depends only on the spot price $S_{t}^{P}$ provided that $S_{t}^{P}<H$, and is independent of the path. It differs from the future value (5.4). In the Black-Scholes world, the UOR option price is (Shreve 2004)

$$
V_{U O R}^{m t m}(t)=\left\{\begin{array}{c}
\phi\left[C_{B S}\left(S_{t}^{P}, K, r, T-t, \sigma_{i m p}\right)-C_{B S}\left(S_{t}^{P}, H, r, T-t, \sigma_{i m p}\right)\right]- \\
\phi\left(\frac{S_{t}^{P}}{H}\right)^{-\frac{2 r}{\sigma_{i m p}^{2}}+1}\left[C_{B S}\left(\frac{H^{2}}{S_{t}^{P}}, K, r, T-t, \sigma_{i m p}\right)-C_{B S}\left(\frac{K H}{S_{t}^{P}}, K, r, T-t, \sigma_{i m p}\right)\right] \\
+R\left[\Phi\left(\frac{\alpha(T-t)-h}{\sqrt{T-t}}\right)+e^{2 \alpha h} \Phi\left(-\frac{\alpha(T-t)+h}{\sqrt{T-t}}\right)\right]
\end{array}\right\}
$$

where $C_{B S}(S, K, r, \tau, \sigma)$ is defined in (4.3), $\alpha=\frac{r}{\sigma_{\text {imp }}}-\frac{\sigma_{i m p}}{2}, h=\frac{1}{\sigma_{i m p}} \ln \left(\frac{H}{S_{t}^{P}}\right)$. The interest rate is given by $r=-\frac{1}{(T-t)} \ln B(t, T)$.

When it comes to modeling barrier option exposure, two issues must be addressed. The first is the determination of the option type at simulation dates. This can partially be resolved by pathwise simulation. If the stock price exceeds the barrier $H$ on any simulation date, the barrier option ceases to exist afterwards. However, stock price monitoring at simulation dates dose not account for the possibility that the stock price may go above and comes back below the barrier between simulation dates, especially when simulation dates are far apart. This leads to the second issue, quantification of the pathwise barrier survival probability, which is the focus of this section. The lack of underlying state variable monitoring is a main reason that pathwise method is preferable to the DJS method for scenario path generation.

The future value of the UOR option is

$$
V_{U O R}(t)=1\left(\tilde{S}_{t}^{P}<H\right) V_{U O R}^{m t m}(t)+1\left(\tilde{S}_{t}^{P} \geq H\right) R B(t, T)
$$

The future value of a today's UOR option is a weighted average of a new UOR option and the rebate depending on whether the stock has hit the barrier by that time. If $1\left(\tilde{S}_{t}^{P}<H\right)=1$, the UOR option survives and the value is given by (5.2). Otherwise, the option has been knocked out and the value is the time t value of the rebate $R$.

Assuming continuous monitoring, the survival indicator $1\left(\tilde{S}_{t}^{P}<H\right)$ takes value 1 with the conditional barrier survival probability $P^{P}\left(\tilde{S}_{t}^{P}<H \mid \mathcal{F}_{t}^{P}\right)$. The superscript $P$ indicates that the probability is under the $P$-measure.

Hence, the future value of the UOR option can be estimated as

$$
V_{U O R}(t)=P^{P}\left(\tilde{S}_{t}^{P}<H \mid \mathcal{F}_{t}^{P}\right) V_{U O R}^{m t m}(t)+P^{P}\left(\tilde{S}_{t}^{P} \geq H \mid \mathcal{F}_{t}^{P}\right) R B(t, T)
$$

The path history $\mathcal{F}_{t}^{P}$ determines the conditional survival probability $P^{P}\left(\tilde{S}_{t}^{P}<H \mid \mathcal{F}_{t}^{P}\right)$. 
Given a simulation date grid $G=\left\{t_{1}, t_{2}, \cdots, t_{L}\right\}$, the stock price at a simulation date $t_{k} \in G$ is known from the stock path simulation. For example, we know the stock price $S_{t_{k}}^{P}(\omega)$ on every simulation date $t_{k}$ of the path $\omega$. If $S_{t_{k}}^{P}(\omega) \geq H$ for some $k$, then $1\left(\tilde{S}_{t}^{P}<H\right)=0$ for $t \geq t_{k}$.

The discrete monitoring of barrier crossing ignores the possibility that the barrier may be crossed between simulation dates. For barrier option exposure calculation, monitoring stock price at the simulation dates is insufficient.

Assuming that the real-world stock price follows a lognormal process

$$
d \ln S_{t}^{P}=\left(\mu^{P}-\frac{1}{2}\left(\sigma^{P}\right)^{2}\right) d t+\sigma^{P} d w_{t}^{P}
$$

Conditional on $S_{t_{k-1}}^{P}(\omega)<H$ and $S_{t_{k}}^{P}(\omega)<H, \ln S_{t}^{P}(\omega)$ is a Brownian bridge for $t_{k-1}<t<t_{k}$. The survival probability is then the probability of the maximum of the Brownian bridge staying below $H$. This conditional probability is (Glasserman 2004)

$$
P^{P}\left(\tilde{S}_{t \in\left(t_{k-1}, t_{k}\right)}^{P}<H \mid S_{t_{k-1}}^{P}<H, S_{t_{k}}^{P}<H\right)=1-\operatorname{Exp}\left(-2 \frac{\ln \left(H / S_{t_{k-1}}^{P}\right) \ln \left(H / S_{t_{k}}^{P}\right)}{\left(\sigma^{P}\right)^{2}\left(t_{k}-t_{k-1}\right)}\right)
$$

As expected, the conditional survival probability decreases as the stock volatility increases since a higher volatility makes barrier crossing more likely. In addition, the closer to $H$ is $S_{t_{k-1}}^{P}$ and/or $S_{t_{k}}^{P}$, the smaller is the conditional survival probability as it is more likely that the stock price crosses the barrier.

The pathwise barrier survival indicator $1\left(\tilde{S}_{t_{k}}^{P}<H\right)$ at the simulation date $t_{k}$ can be calculated recursively by ${ }^{9}$

$$
\begin{aligned}
P^{P}\left(\tilde{S}_{t_{k}}^{P}<H \mid \mathcal{F}_{t_{k}}^{P}\right)=\prod_{j=1}^{k} P^{P}\left(\tilde{S}_{t \in\left(t_{j-1}, t_{j}\right)}^{P}<H \mid S_{t_{j-1}}^{P}<H, S_{t_{j}}^{P}<H\right) 1\left(S_{t_{j}}^{P}<H\right) \\
\quad=P^{P}\left(\tilde{S}_{t_{k-1}}^{P}<H \mid \mathcal{F}_{t_{k-1}}^{P}\right)\left[1-\operatorname{Exp}\left(-2 \frac{\ln \left(H / S_{t_{k-1}}^{P}\right) \ln \left(H / S_{t_{k}}^{P}\right)}{\left(\sigma^{P}\right)^{2}\left(t_{k}-t_{k-1}\right)}\right)\right] 1\left(S_{t_{k}}^{P}<H\right)
\end{aligned}
$$

where, by definition, $1\left(S_{0}^{P}<H\right)=P^{P}\left(\tilde{S}_{0}^{P}<H \mid \mathcal{F}_{0}\right)=1$. Since $1\left(S_{t_{j}}^{P}<H\right)$ is available from the stock price path simulation, we have $P^{P}\left(\tilde{S}_{t_{k}}^{P}<H \mid \mathcal{F}_{t_{k}}^{P}\right)=0$ if $S_{t_{j}}^{P} \geq H$ for some $j \leq k$. Clearly, the pathwise barrier survival probability given in (5.8) is smaller than the DJS-based barrier survival probability which is equal to $\left[1-\operatorname{Exp}\left(-2 \frac{\ln \left(H / S_{0}^{P}\right) \ln \left(H / S_{t_{k}}^{P}\right)}{\left(\sigma^{P}\right)^{2} t_{k}}\right)\right] 1\left(S_{t_{k}}^{P}<H\right)$.

Because the UOR option value (5.4) is always positive, the standalone EE is

$$
E E_{U O R}(t)=E^{P}\left\{1\left(X_{t}^{\operatorname{Max}}<h\right) V_{U O R}^{\text {mtm }}(t) \mid \mathcal{F}_{0}\right\}+R B(t, T) E^{P}\left\{1\left(X_{t}^{\operatorname{Max}} \geq h\right) \mid \mathcal{F}_{0}\right\}
$$

where

\footnotetext{
${ }^{9}$ In the DJS, the barrier survival probability is $P^{P}\left(\tilde{S}_{t_{k}}^{P}<H \mid \mathcal{F}_{t_{k}}^{P}\right)=P^{P}\left(\tilde{S}_{t_{k}}^{P}<H \mid S_{t_{k}}^{P}<H\right) 1\left(S_{t_{k}}^{P}<H\right)$ (Lomibao and Zhu 2006). It is non-zero if $S_{t_{k}}^{P}<H$, whereas (5.8) indicates that this probability is zero if the stock price crosses the barrier at any simulation dates prior to $t_{k}$, ie, if there is $j<k$ such that $S_{t_{j}}^{P} \geq H$.
} 


$$
\left\{\begin{array}{c}
X_{t}=\ln \left(S_{t}^{P} / S_{0}\right) / \sigma^{P}=\alpha t+w_{t}^{P} \\
\alpha=\mu^{P} / \sigma^{P}-\sigma^{P} / 2 \\
h=\ln \left(H / S_{0}\right) / \sigma^{P} \\
X_{t}^{\operatorname{Max}}=\max _{s \leq t} X_{s}
\end{array}\right.
$$

It is well known that the probability of barrier crossing before time $t$ is (Shreve 2004)

$$
E^{P}\left\{1\left(X_{t}^{M a x} \geq h\right) \mid \mathcal{F}_{0}\right\}=\Phi\left(\frac{\alpha t-h}{\sqrt{t}}\right)+e^{2 \alpha h} \Phi\left(-\frac{\alpha t+h}{\sqrt{t}}\right)
$$

The $P$-measure joint density function of $(m, w)=\left(X_{t}^{\operatorname{Max}}, X_{t}\right)$ is (Shreve 2004)

$$
f(m, w)=\frac{2(2 m-w)}{\sqrt{2 \pi t^{3}}} e^{\alpha w-\frac{1}{2} \alpha^{2} t-\frac{1}{2 t}(2 m-w)^{2}}
$$

Recognizing that $V_{U O R}^{m t m}(t)$ depends on $X_{t}=w$ not $X_{t}^{M a x}=m$, and $m \geq w$, we have

$$
E^{P}\left\{1\left(X_{t}^{M a x}<h\right) V_{U O R}^{m t m}(t) \mid \mathcal{F}_{0}\right\}=\left\{\begin{array}{c}
\int_{0}^{h} V_{U O R}^{m t m}(t) d w \int_{w+}^{h} f(m, w) d m \\
+\int_{-\infty}^{0} V_{U O R}^{m t m}(t) d w \int_{0}^{h} f(m, w) d m
\end{array}\right\}
$$

Carrying out the inner integral analytically, we obtain

$$
E^{P}\left\{1\left(X_{t}^{M a x}<h\right) V_{U O R}^{m t m}(t) \mid \mathcal{F}_{0}\right\}=\frac{e^{-\alpha^{2} t / 2}}{\sqrt{2 \pi t}} \int_{-\infty}^{h} V_{U O R}^{m t m}(t) e^{\alpha w}\left[e^{-w^{2} / 2 t}-e^{-(2 h-w)^{2} / 2 t}\right] d w
$$

(5.14) can be calculated on a finite interval because $e^{-w^{2} / 2 t}$ dominates as $w \rightarrow-\infty$.

(5.9), (5.10) and (5.14) form a semi-analytic model for standalone EE of UOR option. The only model dependent part is the scenario model (5.6) which is widely used in the financial industry for stock price scenario generation. (5.14) can be used to benchmark simulation based EE.

Utilizing the following relationships

$$
\left\{\begin{aligned}
1\left(\tilde{S}_{T}<H\right) & =1\left(\tilde{S}_{t}<H\right) 1\left(\tilde{S}_{T}<H\right) \\
1\left(\tilde{S}_{T} \geq H\right) & =1\left(\tilde{S}_{t}<H\right) 1\left(\tilde{S}_{T} \geq H\right)+1\left(\tilde{S}_{t} \geq H\right) \\
D^{Q}(0, T) & =D^{Q}(0, t) D^{Q}(t, T)
\end{aligned}\right.
$$

we obtain

$$
E^{Q}\left\{D^{Q}(0, t) V_{U O R}(t) \mid \mathcal{F}_{0}\right\}=E^{Q}\left\{D^{Q}(0, T) g_{U O R}(T) \mid \mathcal{F}_{0}\right\}=V_{U O R}^{m t m}(0)
$$

where $V_{U O R}^{m t m}(0)$ is the current price of the UOR option which may be calculated using either (5.3) or some other barrier pricing model.

The standalone UCVA of UOR option is

$$
\begin{aligned}
U C V A_{U O R} & =\delta_{C} \int_{0}^{T} E^{Q}\left[D^{Q}(0, t) V_{U O R}(t) \mid \mathcal{F}_{0}\right] d P(t) \\
& =\delta_{C} \int_{0}^{T} V_{U O R}^{m t m}(0) d P(t)=\delta_{C} V_{U O R}^{m t m}(0) P(T)
\end{aligned}
$$


The only condition underlying (5.16) is that the counterparty default is uncorrelated with the UOR option value, ie, no wrong-way-risk.

Remark 5.1: (5.16) indicates that, if $P=Q$ and interest rate is deterministic, the standalone EE of UOR option can be simplified to

$$
E E_{U O R}(t)=B(0, t)^{-1} V_{U O R}^{m t m}(0)
$$

\section{Conclusions}

In this paper, we presented a practical model for estimating the probability that a European swaption is exercised and becomes a swap. The salient point is that the model is consistent with the valuation of the underlying swap in that the factors driving the swaption and the underlying swap are the same short rate, thereby avoiding the need for a separate process as required by the swap rate model of Lomibao and Zhu (2006). Though the model is presented under a one-factor short rate model framework, it could be extended to other one-factor model, such as LMM or HJM model.

We have discussed a practical method to handle the path-dependency in the barrier options. The model can be incorporated into any scenario/revaluation framework for counterparty exposure and CVA. It is more accurate than the one-step simulation counterpart because it takes into account all information available from the path simulation and the probability of barrier crossing is conditioned on the two closest simulation dates.

We have provided analytic formulas for standalone EE, PFE and CVA for swap, European swaption and barrier option. These formulas have practical utility as the Hull-White model is widely used in the financial industry and hence can be used as benchmarks for model validation for Monte Carlo results should converge to the analytic results.

Finally, we have specifically distinguished between pricing and scenario measures, which is one of the crucial differences between CVA and real-world risk measures. Since large scale systems for counterparty credit risk practically require MC simulation, it is important to use a single set of scenarios for both risk factor projection and instrument pricing.

\section{References}

1. Brace, A. and Womersley, R. S. (2000). Exact fit to the swaption volatility matrix using semidefinite programming. Working paper presented at ICBI Global Derivatives Conference, Paris.

2. Brigo, D., Buescu, C. and Morini, M. (2011). Impact of the first to default on bilateral CVA. Working paper.

3. Brigo, D., Morini, M. and Pallavicini, A. (2013). Counterparty Credit Risk, Collateral and Funding. Wiley Finance.

4. Glasserman, P. (2004) Monte Carlo Methods in Financial Engineering. Springer.

5. Hull, J. (1993). Options, Futures, and other Derivative Securities. $2^{\text {nd }}$ Edition. Prentice Hall.

6. Hull, J. and White, A. (1990). Pricing interest-rate derivative securities. The Review of Financial Studies, Vol. 3. No. 4. pp. 573-592.

7. Jamshidian, F. (1989). An exact bond pricing formula. Journal of Finance. 44, pp. 205-209.

8. Lomibao, D. and Zhu, D. (2006). A Conditional Valuation Approach for Path-Dependent Instruments. Wilmott magazine.

9. Shreve, S. E. (2004). Stochastic Calculus for Finance II. Springer.

10. Stein, H. and Lee, K. P. (2010). Counterparty and Credit Risk. Bloomberg. 
11. Zhou, R. (2013). Counterparty Risk Subject to Additional Termination Clauses. Journal of Credit Risk. Vol. 9. No.1. pp. 39-73.

\section{Appendix A: Swap Exposure in Hull-White Model}

Let the interest rate scenarios be generated by the Vasicek model

$$
d r_{t}^{P}=\kappa^{P}\left(\theta^{P}-r_{t}^{P}\right) d t+\sigma^{P} d w_{t}^{P}
$$

The zero coupon bond price defined by (2.2) under the one-factor Hull-White model is

$$
B(t, T)=E^{Q}\left(e^{-\int_{t}^{T} r_{s}^{Q} d s} \mid r_{t}^{P}\right)=e^{A^{Q}(t, T)-C^{Q}(t, T) r_{t}^{P}}
$$

where

$$
\begin{aligned}
& A^{Q}(t, T)=\ln \left(\frac{P(0, T)}{P(0, t)}\right)-C^{Q}(t, T) \frac{\partial \ln P(0, t)}{\partial t}-\frac{\left(\sigma^{Q}\right)^{2}}{4 \kappa^{Q}}\left(1-e^{-2 \kappa^{Q} t}\right) C^{Q}(t, T)^{2} \\
& C^{Q}(t, T)=\left(1-e^{-\kappa^{Q}(T-t)}\right) / \kappa^{Q}
\end{aligned}
$$

The pricing model parameters are calibrated to the initial yield curve $P(0, t)$ and the initial option market.

By virtue of (2.3), the forward swap EE is

$$
E E_{\text {FwdSwap }}(t)=E^{P}\left\{B\left(t, T_{0}\right)\left[1-\frac{B\left(t, T_{N}\right)}{B\left(t, T_{0}\right)}-K \sum_{m=1}^{M} \frac{B\left(t, S_{m}\right)}{B\left(t, T_{0}\right)} \Delta S_{m-1}\right]^{+} \mid \mathcal{F}_{0}\right\}
$$

Since for $T>T_{0}, \frac{B(t, T)}{B\left(t, T_{0}\right)}$ decreases with increasing $r_{t}^{P}$, there exists a critical value $r_{c}(t)$ such that (Jamshidian 1989)

$$
\left.\left\{1-\frac{B\left(t, T_{N}\right)}{B\left(t, T_{0}\right)}-K \sum_{m=1}^{M} \frac{B\left(t, S_{m}\right)}{B\left(t, T_{0}\right)} \Delta S_{m-1}\right\}\right|_{r_{t}^{P}=r_{c}}=0
$$

The critical value $r_{c}$ requires numerical procedure but is a deterministic function of t. Clearly, only part of the distribution where $r_{t}^{P}>r_{c}(t)$ contributes to the EE. This is expected for a payer swap where an increase in interest rate benefits the fixed rate payer. The interest rate effect is reversed for a receiver swap where the EE contribution comes from $r_{t}^{P}<r_{c}$. Having found the critical rate $r_{c}$, (A.5) becomes

$$
E E_{\text {FwdSwap }}\left(t ; r_{t}^{P}\right)=E^{P}\left\{\left[B\left(t, T_{0}\right)-B\left(t, T_{N}\right)-K \sum_{m=1}^{M} B\left(t, S_{m}\right) \Delta S_{m-1}\right] 1\left(r_{t}^{P}>r_{c}\right) \mid \mathcal{F}_{0}\right\}
$$

where

$$
E^{P}\left\{B(t, T) 1\left(r_{t}^{P}>r_{c}\right) \mid \mathcal{F}_{0}\right\}=\left\{\begin{array}{c}
e^{A^{Q}(t, T)-C^{Q}(t, T) \mu_{e}(t)+\frac{1}{2}\left(C^{Q}(t, T) \sigma_{e}(t)\right)^{2}} \\
\times \Phi\left(-\frac{r_{c}(t)-\mu_{e}(t)}{\sigma_{e}(t)}-C^{Q}(t, T) \sigma_{e}(t)\right)
\end{array}\right\}
$$

for $T=T_{0}, T_{N}, S_{m}, m=1, \cdots, M$, and $r_{t}^{P}=\mu_{e}(t)+\sigma_{e}(t) \epsilon$ with

$$
\mu_{e}(t)=r_{0}^{P} e^{-\kappa^{P} t}+\theta^{P}\left(1-e^{-\kappa^{P} t}\right), \sigma_{e}(t)=\sigma^{P} \sqrt{\left(1-e^{-2 \kappa^{P} t}\right) / 2 \kappa^{P}} .
$$


The PFE for the forward swap can be also evaluated analytically. It can be shown that the forward payer swap value increases with increasing $r_{t}^{P}$, ie, $\frac{\partial V_{F w d S w a p}(t)}{\partial r_{t}^{P}}>0$. This indicates that the PFE corresponds to the $\alpha$-percentile of the $r_{t}^{P}$-distribution conditional. In other words, the PFE is

$$
P F E_{F w d S w a p}(t)=\operatorname{Max}\left\{V_{F w d S w a p}\left(t ; r_{t}^{P}(\alpha)\right), 0\right\}
$$

where $r_{t}^{P}(\alpha)=\mu_{e}(t)+\sigma_{e}(t) \Phi^{-1}(\alpha), \Phi^{-1}(\alpha)$ is the inverse standard cumulative normal distribution function.

Tail swap exposure is more involved due to the presence of stochastic accrued interest. The tail swap EE is given by

$$
E E_{\text {Tailswap }}(t)=\iint_{-\infty}^{\infty}\left[V_{\text {Tailswap }}(t)\right]^{+} \phi_{2}(x, y ; \rho) d x d y
$$

where $\phi_{2}(x, y, \rho)$ is the standard bivariate normal density function, and the correlation is

$$
\rho=\operatorname{Corr}\left(r_{t}^{P}, r_{T_{\alpha(t)-1}^{P}}\right)=\frac{\left.\left.e^{-\kappa^{P}(t-T \alpha(t)-1}\right)_{-e^{-\kappa^{P}(t+T} \alpha(t)-1}\right)}{\sqrt{1-e^{-2 \kappa^{P} t}} \sqrt{1-e^{-2 \kappa^{P} T_{\alpha(t)-1}}}}
$$

The zero-coupon bond prices in the tail swap formula (3.1) are

$$
\begin{aligned}
& B\left(T_{\alpha(t)-1}, T_{\alpha(t)}\right)=e^{A^{Q}\left(T_{\alpha(t)-1}, T_{\alpha(t)}\right)-C^{Q}\left(T_{\alpha(t)-1}, T_{\alpha(t)}\right) r_{T}^{P}}{ }_{\alpha(t)-1} \\
& B(t, T)=e^{A^{Q}(t, T)-C^{Q}(t, T) r_{t}^{P}}, T=T_{\alpha(t)}, T_{N}, S_{\beta(t)}, S_{\beta(t)+1}, \cdots, S_{M}
\end{aligned}
$$

with $r_{t}^{P}=\mu_{e}(t)+\sigma_{e}(t) x$ and $r_{T_{\alpha(t)-1}}^{P}=\mu_{e}\left(T_{\alpha(t)-1}\right)+\sigma_{e}\left(T_{\alpha(t)-1}\right) y$.

Given $r_{t}^{P}$ or equivalently $x, V_{\text {Tailswap }}(t)$ is an increasing function of $r_{\alpha(t)-1}^{P}$ or $y$. This means that $V_{\text {Tailswap }}(t)>0$ iff $y>y_{c}(x)$ where

$$
y_{c}(x)=\frac{A^{Q}\left(T_{\alpha(t)-1}, T_{\alpha(t)}\right)+\ln \left(E(x) / B\left(t, T_{\alpha(t)}\right)\right)}{C^{Q}\left(T_{\alpha(t)-1}, T_{\alpha(t)}\right) \sigma_{e}\left(T_{\alpha(t)-1}\right)}-\frac{\mu_{e}\left(T_{\alpha(t)-1}\right)}{\sigma_{e}\left(T_{\alpha(t)-1}\right)}
$$

with $E(x)$ defined in (A.18) below.

The right-hand-side of (A.15) is a decreasing function of $x$. The higher is the $r_{t}^{P}$, the lower is the threshold $y_{c}(x)$ such that $V_{\text {Tailswap }}(t)=0$. As a result, (A.11) becomes

$$
E_{\text {Tailswap }}(t)=\int_{-\infty}^{\infty}\left\{\int_{y_{c}}^{\infty} V_{\text {Tailswap }}(t) \phi_{2}(x, y ; \rho) d y\right\} d x
$$

Since only $B\left(T_{\alpha(t)-1}, T_{\alpha(t)}\right)$ depends on $y$, we integrate with respect to $y$ to yield 


$$
\int_{y_{c}}^{\infty} V_{\text {TailSwap }}(t) \phi_{2}(x, y ; \rho) d y=\left\{\begin{array}{c}
B\left(t, T_{\alpha(t)}\right) e^{b+a \rho x} \Phi\left(a \sqrt{1-\rho^{2}}+\frac{\rho x-y_{c}(x)}{\sqrt{1-\rho^{2}}}\right) \phi(x) \\
-E(x) \Phi\left(\frac{\rho x-y_{c}(x)}{\sqrt{1-\rho^{2}}}\right) \phi(x)
\end{array}\right\}
$$

where

$$
\left\{\begin{array}{l}
a=C^{Q}\left(T_{\alpha(t)-1}, T_{\alpha(t)}\right) \sigma_{e}\left(T_{\alpha(t)-1}\right) \\
c=-A^{Q}\left(T_{\alpha(t)-1}, T_{\alpha(t)}\right)+C^{Q}\left(T_{\alpha(t)-1}, T_{\alpha(t)}\right) \mu_{e}\left(T_{\alpha(t)-1}\right) \\
b=c+\frac{1}{2} a^{2}\left(1-\rho^{2}\right) \\
E(x)=B\left(t, T_{N}\right)+K \sum_{m=\beta(t)}^{M} B\left(t, S_{m}\right) \Delta S_{m-1} \\
B(t, T)=e^{A^{Q}(t, T)-C^{Q}(t, T) \mu_{e}(t)-C^{Q}(t, T) \sigma_{e}(t) x}
\end{array}\right.
$$

Using (A.15) to (A.18), we obtain

$$
E P E_{\text {Tailswap }}(t)=\int_{-\infty}^{\infty}\left\{B\left(t, T_{\alpha(t)}\right) e^{b+a \rho x} \Phi\left(a \sqrt{1-\rho^{2}}+\frac{\rho x-y_{c}(x)}{\sqrt{1-\rho^{2}}}\right)-E(x) \Phi\left(\frac{\rho x-y_{c}(x)}{\sqrt{1-\rho^{2}}}\right)\right\} \phi(x) d x
$$

with

$$
\begin{aligned}
& \int_{-\infty}^{\infty} B\left(t, T_{\alpha(t)}\right) e^{b+a \rho x} \Phi\left(a \sqrt{1-\rho^{2}}+\frac{\rho x-y_{c}(x)}{\sqrt{1-\rho^{2}}}\right) \phi(x) d x=D \int_{-\infty}^{\infty} \Phi\left(\frac{\rho x-y_{c}}{\sqrt{1-\rho^{2}}}+I\right) \phi(x) d x \\
& \int_{-\infty}^{\infty} B(t, T) \Phi\left(\frac{\rho x-y_{c}(x)}{\sqrt{1-\rho^{2}}}\right) \phi(x) d x=G(T) \int_{-\infty}^{\infty} \Phi\left(\frac{\rho x-y_{c}(x)}{\sqrt{1-\rho^{2}}}+H(T)\right) \phi(x) d x
\end{aligned}
$$

where

$$
\left\{\begin{array}{l}
\left.\left.D=e^{A^{Q}\left(t, T_{\alpha(t)}\right)-C^{Q}\left(t, T_{\alpha(t)}\right) \mu_{e}(t)+b+\frac{1}{2}\left(C^{Q}(t, T\right.} T_{\alpha(t)}\right) \sigma_{e}(t)\right)^{2} \\
I=a \sqrt{1-\rho^{2}}-\frac{\rho C^{Q}(t, T \alpha(t)) \sigma_{e}(t)}{\sqrt{1-\rho^{2}}} \\
G(T)=e^{A^{Q}(t, T)-C^{Q}(t, T) \mu_{e}(t)+\frac{1}{2}\left(C^{Q}(t, T) \sigma_{e}(t)\right)^{2}} \\
H(T)=-\frac{\rho C^{Q}(t, T) \sigma_{e}(t)}{\sqrt{1-\rho^{2}}}
\end{array}\right.
$$

Thus, computation of $E P E_{\text {Tailswap }}(t)$ amounts to computing the integrals

$$
\int_{-\infty}^{\infty} \Phi\left(\frac{\rho x-y_{c}(x)}{\sqrt{1-\rho^{2}}}+A\right) \phi(x) d x=\int_{-d}^{d} \Phi\left(\frac{\rho x-y_{c}(x)}{\sqrt{1-\rho^{2}}}+A\right) \phi(x) d x+e r r
$$

with $A=I, H(T), T=T_{N}, S_{m}, m=\beta(t), \cdots, M$. Since $0<\Phi\left(\frac{\rho x-y_{c}(x)}{\sqrt{1-\rho^{2}}}+A\right)<1$, the error can be made arbitrarily small, $0<$ err $<2 \Phi(-d)$. For example, $2 \Phi(-3) \approx 0.003$.

Substituting all these into (A.16) results in 


$$
E E_{\text {Tailswap }}(t)=\left\{\begin{array}{c}
D E\left\{\Phi\left(\frac{\rho x-y_{c}(x)}{\sqrt{1-\rho^{2}}}+I\right)\right\}-\mathrm{G}\left(T_{N}\right) E\left\{\Phi\left(\frac{\rho x-y_{c}(x)}{\sqrt{1-\rho^{2}}}+H\left(T_{N}\right)\right)\right\} \\
-K \sum_{m=1}^{M} \Delta S_{m-1} \mathrm{G}\left(S_{m}\right) E\left\{\Phi\left(\frac{\rho x-y_{c}(x)}{\sqrt{1-\rho^{2}}}+H\left(S_{m}\right)\right)\right\}
\end{array}\right\}
$$

We now describe a model for the swaption PFE at $t>T_{0}$. From (2.4), we have

$$
P^{P}\left\{V_{\text {Tailswap }}(t) \geq P F E_{\alpha}(t) \mid \mathcal{F}_{0}\right\}=E^{P}\left\{P^{P}\left[V_{\text {Tailswap }}(t) \geq P F E_{\alpha}(t) \mid x\right]\right\}=1-\alpha
$$

By virtue of (A.18), we have the set relation

$$
\left\{V_{\text {TailSwap }}(t) \geq P F E_{\alpha}(t)\right\}=\left\{y \geq \frac{1}{a}\left[\ln \left(\frac{P F E_{\alpha}(t)+E(x)}{B(t, T \alpha(t)}\right)-c\right]\right\}
$$

Using the conditional distribution $y \mid x \sim \Phi\left(\rho x, 1-\rho^{2}\right)$, we have

$$
\begin{aligned}
P^{P}\left[V_{\text {Tailswap }}(t) \geq P F E_{\alpha}(t) \mid x\right] & =P^{P}\left\{y \geq \frac{1}{a}\left[\ln \left(\frac{P F E_{\alpha}(t)+E(x)}{B\left(t, T_{\alpha(t)}\right)}\right)-c\right] \mid x\right\} \\
& =\Phi\left(\frac{\rho x+\frac{c}{a}-\frac{1}{a} \ln \left(\frac{P F E_{\alpha}(t)+E(x)}{B\left(t, T_{\alpha(t)}\right)}\right)}{\sqrt{1-\rho^{2}}}\right)
\end{aligned}
$$

Substituting (A.22) into (A.21), we see that $P F E_{\alpha}(t)$ is the solution of the integral equation

$$
\int_{-\infty}^{\infty} \Phi\left(\frac{\rho x+\frac{c}{a}-\frac{1}{a} \ln \left(\frac{P F E_{\alpha}(t)+E(x)}{B(t, T \alpha(t)}\right)}{\sqrt{1-\rho^{2}}}\right) \phi(x) d x=1-\alpha
$$

where $E(x), a$ and $c$ are given in (A.18), and $B\left(t, T_{\alpha(t)}\right)$ is given in (A.14). If $P F E_{\alpha}(t)<0$ then set $P F E_{\alpha}(t)=0$.

We now discuss an analytic model for swaption EE at $t>T_{0}$. In section 2.3, we show that there is a critical short rate $r_{C}$ such that $V_{F w d S w a p}\left(T_{0}\right)>0$ iff $r_{T_{0}}^{P}>r_{c}$. Following the same argument, the swaption EE at $t>T_{0}$ can be casted as

$$
E^{P}\left[V_{\text {Tailswap }}(t)^{+} 1\left(V_{\text {FwdSwap }}\left(T_{0}\right)>0\right) \mid \mathcal{F}_{0}\right]=\int_{-\infty}^{\infty} \int_{y_{c}}^{\infty} V_{\text {Tailswap }}(t) d y d x \int_{z_{c}}^{\infty} \phi_{3}(x, y, z) d z
$$

where $z_{c}=\left(r_{c}-\mu_{e}\left(T_{0}\right)\right) / \sigma_{e}\left(T_{0}\right)$ is independent of both $x$ and $y$.

In (A.29), $\phi_{3}(x, y, z)$ is the joint three-dimensional standard normal density function

$$
\phi_{3}(x, y, z ; R)=\frac{1}{(2 \pi)^{3 / 2}(\operatorname{det}(R))^{1 / 2}} \operatorname{Exp}\left\{-\frac{1}{2}(x, y, z) \mathrm{R}^{-1}(x, y, z)^{T}\right\}
$$

where correlation matrix is 


$$
\mathrm{R}=\left(\begin{array}{ccc}
1 & \rho_{t, T_{\alpha(t)-1}} & \rho_{t, T_{0}} \\
\rho_{t, T_{\alpha(t)-1}} & 1 & \rho_{T_{0}, T_{\alpha(t)-1}} \\
\rho_{t, T_{0}} & \rho_{T_{0}, T_{\alpha(t)-1}} & 1
\end{array}\right)=\left(\begin{array}{ccc}
1 & u_{12} & u_{13} \\
u_{12} & 1 & u_{23} \\
u_{13} & u_{23} & 1
\end{array}\right)=\left(\begin{array}{cc}
A_{2 \times 2} & V_{2 \times 1} \\
V^{T} & 1
\end{array}\right)
$$

with $\rho_{u, v}=\operatorname{Corr}\left(r_{u}^{P}, r_{v}^{P}\right)=\frac{e^{-\kappa^{P}(\max (u, v)-\min (u, v))}-e^{-\kappa^{P}(u+v)}}{\sqrt{1-e^{-2 \kappa^{P} u}} \sqrt{1-e^{-2 \kappa^{P} v}}}$, particularly, $u_{12}=\rho$ defined in (A.12).

Similar to (A.16), we can reduce (A.29) to a 1D integration. To this end, we first obtain

$$
\int_{z_{c}}^{\infty} \phi_{3}(x, y, z ; R) d z=\phi_{2}(x, y ; \rho) \Phi\left(-\frac{w_{1} x+w_{2} y+z_{c}}{\sqrt{k}}\right)=\phi(x) \Phi\left(-\frac{w_{1} x+w_{2} y+z_{c}}{\sqrt{k}}\right) \phi_{y \mid x}(y)
$$

where $\phi_{y \mid x}(y)=\frac{1}{\sqrt{2 \pi\left(1-\rho^{2}\right)}} e^{-\frac{1(y-\rho x)^{2}}{21-\rho^{2}}}$ is the conditional density function.

The parameters are defined as

$$
\left\{\begin{array}{c}
R^{-1}=\left(\begin{array}{cc}
U & W \\
W^{T} & 1 / k
\end{array}\right) \\
\operatorname{det}(\mathrm{R})=1-u_{12}^{2}-u_{13}^{2}-u_{23}^{2}+2 u_{12} u_{13} u_{23} \\
U=A^{-1}+\frac{1}{k} A^{-1} V\left(A^{-1} V\right)^{T} \\
W=\left(\begin{array}{l}
w_{1} \\
w_{2}
\end{array}\right)=-\frac{A^{-1} V}{k}=-\frac{k^{-1}}{1-u_{12}^{2}}\left(\begin{array}{l}
u_{13}-u_{12} u_{23} \\
u_{23}-u_{12} u_{13}
\end{array}\right) \\
k=1-V^{T} A^{-1} V=\frac{\operatorname{det}(R)}{1-u_{12}^{2}} \\
A^{-1}=\frac{1}{1-u_{12}^{2}}\left(\begin{array}{cc}
1 & -u_{12} \\
-u_{12} & 1
\end{array}\right)=\frac{1}{1-\rho^{2}}\left(\begin{array}{cc}
1 & -\rho \\
-\rho & 1
\end{array}\right)
\end{array}\right.
$$

Defining the function

$$
\begin{aligned}
g(x ; a) & =\int_{y_{c}(x)}^{\infty} e^{a y} \Phi\left(-\frac{w_{1} x+w_{2} y+z_{c}}{\sqrt{k}}\right) \phi_{y \mid x}(y) d y \\
& =e^{\frac{1}{2} a^{2}\left(1-\rho^{2}\right)} \int_{\bar{y}_{c}(x)}^{\infty} \Phi\left(-\frac{\left(w_{1}+\rho w_{2}\right) x+w_{2} \sqrt{1-\rho^{2}} y+z_{c}}{\sqrt{k}}-\frac{z_{c}+a\left(1-\rho^{2}\right) w_{2}}{\sqrt{k}}\right) \phi(y) d y
\end{aligned}
$$

where $\bar{y}_{c}(x)=\frac{y_{c}(x)-\rho x}{\sqrt{1-\rho^{2}}}-a \sqrt{1-\rho^{2}}$, the swaption EE at $t>T_{0}$ can be expressed as

$$
\begin{aligned}
E E_{\text {Swpn }}\left(t>T_{0}\right) & =E^{P}\left[V_{\text {Tailswap }}(t)^{+} 1\left(V_{\text {FwdSwap }}\left(T_{0}\right)>0\right) \mid \mathcal{F}_{0}\right] \\
& =\int_{-\infty}^{\infty}\left\{e^{c} B\left(t, T_{\alpha(t)}\right) g(x ; a)-E(x) g(x ; 0)\right\} \phi(x) d x
\end{aligned}
$$

where the parameters $a$ and $c$ are defined in (A.18).

Remark A: The above results may help explain the potential complexity of Bermudan swaption exposure modeling. Suppose we have a swap-settled swaption where it is automatically exercised on two future dates, $O_{1}$ and $O_{2}$. The option expiry date is $O_{2}$. If the swap value is not positive on $O_{1}$, the swaption continues. Clearly, the swaption EE at $t>O_{2}$ involves 4 correlated interest rates 
at $t, O_{1}, O_{2}, T_{\alpha(t)-1}$. Even without the optimal exercise, the dimensionality of the problem increases as the number of exercise dates. The optimal exercise feature further requires look back.

\section{Appendix B: $B\left(T_{\alpha(t)-1}, T_{\alpha(t)}\right)$ conditional on $\mathcal{F}_{t}^{P}$}

The tail swap value (3.1) depends on $B\left(T_{\alpha(t)-1}, T_{\alpha(t)}\right)$ which may not be available since $T_{\alpha(t)-1}$ may not be a simulation node. In this section, we discuss a model for $B\left(T_{\alpha(t)-1}, T_{\alpha(t)}\right)$ given in (A.13).

Let $h_{k-1}$ and $h_{k}$ be the simulation dates closest to $T_{\alpha(t)-1}$ such that $h_{k-1} \leq T_{\alpha(t)-1}<h_{k} \leq t$, then conditional on $r_{h_{k-1}}^{P}$ and $r_{h_{k}}^{P}$, we simulate (A.13). The value of this conditional sampling is

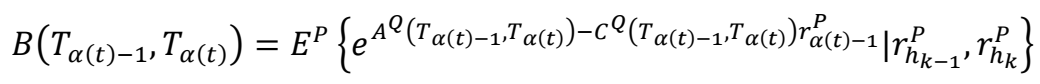

Again, the scenario short rate $r_{\alpha(t)-1}^{P}$ can be modeled as a Brownian bridge. Setting $u=h_{k-1}, v=$ $h_{k}$ and $f(x)=x$ in (C.12), we have

$$
\left(r_{T_{\alpha(t)-1}}^{P} \mid r_{h_{k-1}}^{P}, r_{h_{k}}^{P}\right) \sim \Phi\left(\mu_{T_{\alpha(t)-1}}^{B B},\left(\sigma_{T_{\alpha(t)-1}^{B B}}^{B B}\right)^{2}\right)
$$

where

$$
\left\{\begin{array}{c}
\mu_{T_{\alpha(t)-1}^{B B}}^{B B}=p_{T_{\alpha(t)-1}} r_{h_{k-1}}^{P}+q_{T_{\alpha(t)-1}} r_{h_{k-1}}^{P}+\tilde{\mu}_{T_{\alpha(t)-1}} \\
\sigma_{T_{\alpha(t)-1} B B}^{B B}
\end{array}\right.
$$

Substitution of (B.2) into (B.1) results in

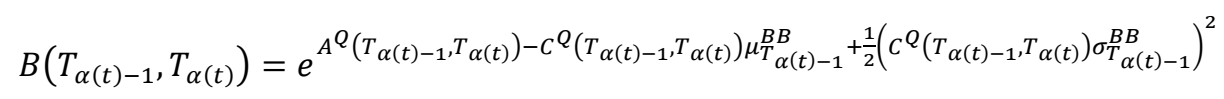

\section{Appendix C: European Swaption Exercise Probability}

We provide derivation for the European swaption exercise probability (4.7) where the scenario short rate evolution is governed by (4.5). To lighten notation, we drop the superscript $P$, and all variables are understood to be under the $P$-measure.

Let $x_{t}=f\left(r_{t}\right)$, then $(4.5)$ gives

$$
x_{t}=x_{0} e^{-k t}+\theta\left(1-e^{-k t}\right)+\int_{0}^{t} \sigma e^{-k(t-s)} d w_{s}=\mu_{t}+\sigma_{t} \epsilon
$$

where $\epsilon$ is a standard normal random number and

$$
\mu_{t}=x_{0} e^{-k t}+\theta\left(1-e^{-k t}\right), \sigma_{t}=\sigma \sqrt{\frac{1-e^{-2 k t}}{2 k}}
$$

The covariance function is

$$
\sigma_{s t}=\operatorname{Cov}\left(x_{s}, x_{t}\right)=\frac{\sigma^{2}}{2 k}\left(e^{-k(\max (s, t)-\min (s, t))}-e^{-k(s+t)}\right)=\sigma_{t s}
$$


(C.3) shows that $x_{t}, x_{u}$ and $x_{v}$ are joint normal with the covariance matrix

$$
\Sigma=\left(\begin{array}{lll}
\sigma_{t t} & \sigma_{t u} & \sigma_{t v} \\
\sigma_{u t} & \sigma_{u u} & \sigma_{u v} \\
\sigma_{v t} & \sigma_{v u} & \sigma_{v v}
\end{array}\right)=\left(\begin{array}{cc}
\sigma_{t t} & \Sigma_{12} \\
\Sigma_{21} & \Sigma_{22}
\end{array}\right), \Sigma_{22}=\left(\begin{array}{cc}
\sigma_{u u} & \sigma_{u v} \\
\sigma_{v u} & \sigma_{v v}
\end{array}\right), \Sigma_{12}=\left(\begin{array}{ll}
\sigma_{t u} & \sigma_{t v}
\end{array}\right), \Sigma_{21}=\left(\Sigma_{12}\right)^{T}
$$

The conditional distribution of $x_{t}$ is (Glasserman 2004)

$$
\left(x_{t} \mid x_{u}, x_{v}\right) \sim \Phi\left(\mu_{t}+\Sigma_{12} \Sigma_{22}^{-1}\left(\begin{array}{c}
x_{u}-\mu_{u} \\
x_{v}-\mu_{v}
\end{array}\right), \sigma_{t t}-\Sigma_{12} \Sigma_{22}^{-1} \Sigma_{21}\right)
$$

where

$$
\begin{aligned}
& \left(\sigma_{t}^{B B}\right)^{2}=\sigma_{t t}-\Sigma_{12} \Sigma_{22}{ }^{-1} \Sigma_{21}=\sigma_{t t}-\left(p_{t} \sigma_{u t}+q_{t} \sigma_{v t}\right) \\
& \mu_{t}^{B B}=\mu_{t}+\Sigma_{12} \Sigma_{22}^{-1}\left(\begin{array}{l}
x_{u}-\mu_{u} \\
x_{v}-\mu_{v}
\end{array}\right)=p_{t} x_{u}+q_{t} x_{v}+\mu_{t}-\left(p_{t} \mu_{u}+q_{t} \mu_{v}\right) \\
& \operatorname{det}\left(\Sigma_{22}\right)=\sigma_{u u} \sigma_{v v}-\sigma_{u v} \sigma_{v u} \\
& p_{t}=\left(\sigma_{t u} \sigma_{v v}-\sigma_{t v} \sigma_{v u}\right) / \operatorname{det}\left(\Sigma_{22}\right) \\
& q_{t}=\left(\sigma_{t v} \sigma_{u u}-\sigma_{t u} \sigma_{u v}\right) / \operatorname{det}\left(\Sigma_{22}\right)
\end{aligned}
$$

Since $p_{u}=1, q_{u}=0, p_{v}=0, q_{v}=1$, we obtain $\mu_{u}^{B B}=x_{u}, \mu_{v}^{B B}=x_{v}, \sigma_{u}^{B B}=\sigma_{v}^{B B}=0$. This means that (C.4) indeed defines a Brownian bridge.

For standard Brownian motion with constant drift, the Brownian bridge has a linear drift and a quadratic variance (Shreve 2004). In this case, the Brownian bridge may be considered as an interpolation between the two endpoints. However, these do not hold for (4.5) as stated in section 4. First, the mean function $\mu_{t}^{B B}$ is not a linear interpolation of the endpoints. The residual term in (C.6) is not zero, ie, $\mu_{t}-\left(p_{t} \mu_{u}+q_{t} \mu_{v}\right) \neq 0$. Second, the variance function $\left(\sigma_{t}^{B B}\right)^{2}$ is much more complex than a quadratic function. It can be shown that when $k \rightarrow 0, \mu_{t}^{B B}$ approaches a linear function and $\left(\sigma_{t}^{B B}\right)^{2}$ approaches a quadratic function.

It can be shown that

$$
\tilde{\mu}_{t}=\mu_{t}-\left(p_{t} \mu_{u}+q_{t} \mu_{v}\right)=\theta\left(1-p_{t}-q_{t}\right)
$$

Thus, it is more instructive to rewrite (C.6) with $\tilde{\mu}_{t}$ being equal to

$$
\mu_{t}^{B B}=p_{t} x_{u}+q_{t} x_{v}+\tilde{\mu}_{t}
$$

The correction term $\tilde{\mu}_{t}$ represents a convexity adjustment due to mean reversion of the Brownian motion. Thus, for mean reversion process, the reversion speed has an impact on the BB's mean. (C.10) shows that the mean-reversion level also affects the mean function. The initial value, however, has no bearing on the Brownian bridge.

Based on (C.5) to (C.11), (C.4) becomes

$$
\left(x_{t} \mid x_{u}, x_{v}\right) \sim \Phi\left(p_{t} x_{u}+q_{t} x_{v}+\tilde{\mu}_{t},\left(\sigma_{t}^{B B}\right)^{2}\right)
$$

Let $x_{c}=f\left(r_{c}\right)$ where $r_{c}$ is the critical rate such that $V_{F w d s w a p}\left(T_{0}\right)>0$. As explained in section 2.3, this is equivalent to $\left(x_{T_{0}} \mid x_{h_{k-1}}, x_{h_{k}}\right)>x_{c}$. By virtue of (C.12) and $x_{T_{0}}=f\left(r_{T_{0}}^{P}\right)$, we obtain 


$$
\begin{aligned}
P^{P}\left(V_{\text {FwdSwap }}\left(T_{0}\right)>0 \mid \mathcal{F}_{h_{k}}^{P}\right) & =P^{P}\left(f\left(r_{T_{0}}^{P}\right)>f\left(r_{c}\right) \mid f\left(r_{h_{k}}^{P}\right), f\left(r_{h_{k-1}}^{P}\right)\right) \\
& =\Phi\left(\left\{f\left(r_{h_{k-1}}^{P}\right) p_{T_{0}}+f\left(r_{h_{k}}^{P}\right) q_{T_{0}}+\tilde{\mu}_{T_{0}}-f\left(r_{c}\right)\right\} / \sigma_{T_{0}}^{B B}\right)
\end{aligned}
$$

This completes the derivation of (4.7). 